\title{
Investigation of Self-Assembly of Two-Component Organogel System Based on Trigonal Acids and Aminobenzothiazole Derivatives
}

\author{
Youbo Di, Wei Hong, and Jinming Dai \\ College of Textile Engineering and Art, Taiyuan University of Technology, Taiyuan 030024, China \\ Correspondence should be addressed to Jinming Dai; jinmingdai12@126.com
}

Received 30 April 2013; Accepted 31 May 2013

Academic Editor: Tifeng Jiao

Copyright (C) 2013 Youbo Di et al. This is an open access article distributed under the Creative Commons Attribution License, which permits unrestricted use, distribution, and reproduction in any medium, provided the original work is properly cited.

\begin{abstract}
We reported here the gelation behaviors of two-component organogel system based on different acids and aminobenzothiazole derivatives in various organic solvents. Their gelation behaviors in 20 solvents were tested as new organic gelators. It was shown that the molecular skeletons and substituted groups in these compounds played a crucial role in the gelation behavior of the mixtures. Only the binary mixture of 2-aminobenzothiazole and trigonal 1,3,5-benzenetricarboxylic acid with aromatic core could form organogels in ethanol and acetone. Morphological observations reveal that the microstructures of both xerogels showed similar wrinkle-shaped domains composed of sheet-like aggregates with many holes. Spectral studies reveal the hydrogen bonding interaction between the amide of the gelator and lamellar-like structure of the aggregates in both gels. The present investigation is a perspective to provide new clues for the design of new nanomaterials and functional textile materials with special microstructures.
\end{abstract}

\section{Introduction}

The self-assembly of small functional molecules into supramolecular structures is a powerful approach to the development of new materials and nanoscale devices [1-4]. The properties of these materials depend not only on the nature of their molecular constituents but also on the precise spatial positioning of the functional groups $[5,6]$. As a novel class of self-assembled materials, low-molecular-mass organic gels (LMOGs) have become one of the most active frontiers in recent years because they can organize into regular nanoarchitectures through specific noncovalent interactions including hydrogen bonds, $\pi-\pi$ interactions, hydrophobic interactions, and other forces [7-10]. Nowadays, physical gelation of organic solvents by LMOGs has become one of the hot areas in soft matter research due to their scientific values and many potential applications in biomedical field, including multifunctional textile materials, drug release, and implants [11-13]. The noncovalent nature of the three-dimensional networks within the supramolecular gels promises accessibility for designing and constructing new textile materials and other molecular devices [14-17]. In addition, most of the gelators reported so far are either serendipitous or derivatives from a well-known gelator, and in most cases, a detailed description of the supramolecular aggregates responsible for gel formation is not investigated due to the intrinsic difficulty of analyzing the superstructure within a gel. Recently, Tang and coworkers reported the utilization of the complementary carboxyl and pyridyl groups in preparing a new kind of gelators [18-20]. For example, they described the preliminary result of the hydrogel based on the complex formed from para-hydroxy pyridinium salt of 1,2,4,5-benzene tetracarboxylic acid [18] and investigated the structure-property relation of hydrogels based on pyridinyl amino butanoic acid isomeric compounds [19].

In this study, we reported the gelation behaviors of twocomponent organogel system based on different acids and aminobenzothiazole derivatives in various organic solvents. Their gelation behaviors in 20 solvents were tested as new organic gelators. We found that the self-assembly of these two-component mixtures can be controlled and directed to form sheet-like supramolecular structures. The detailed morphologies and assembly modes of gels were investigated by scanning electron microscopy (SEM), Fourier transform 
TABLE 1: Gelation behaviors at room temperature ${ }^{\mathrm{a}}$.

\begin{tabular}{lcccccc}
\hline Solvents & SC2 & SC3 & SN3 & NC2 & NC3 & NN3 \\
\hline Cyclopentanone & PS & PS & PS & PS & PS & PS \\
DMF & S & S & S & S & PS & PS \\
1,4-Dioxane & PS & I & PS & PS & PS & S \\
Petroleum ether & I & PS & I & I & I & I \\
Pyridine & S & S & PS & PS & PS & S \\
Ethyl acetate & I & PS & PS & I & PS & PS \\
n-Butanol & I & I & PS & PS & PS & PS \\
Chloroform & PS & PS & S & S & S & PS \\
n-Hexane & I & PS & PS & I & PS & PS \\
Aniline & PS & S & S & PS & S & S \\
Nitrobenzene & PS & PS & PS & PS & PS & S \\
n-Butyl acrylate & I & PS & PS & PS & PS & PS \\
Toluene & I & PS & I & I & I & I \\
Benzene & I & PS & PS & PS & PS & PS \\
THF & PS & PS & PS & PS & PS & S \\
Acetone & PS & G(2.5) & S & PS & S & S \\
Ethanol & PS & G(2.5) & PS & PS & PS & PS \\
Ethanolamine & PD & S & S & I & S & PS \\
Cyclohexanone & PS & PS & S & I & S & PS \\
DMSO & PS & S & S & PS & S & S \\
\hline
\end{tabular}

${ }^{a}$ S: solution; PS: partially soluble; G: gel; I: insoluble; for gels, the critical gelation concentrations at room temperature are shown in parentheses $(\% \mathrm{w} / \mathrm{v})$.

infrared spectrometer (FT-IR), and X-ray diffraction (XRD) methods. This report may afford a specific approach to understanding the soft matter and design of new nanomaterials and functional textile materials.

\section{Experimental Section}

2.1. Reagents and Materials. The starting materials, 2aminobenzothiazole (98\%), 2-aminobenzimidazole (97+\%), 1,3,5-benzenetricarboxylic acid (98\%), nitrilotriacetic acid (99\%), and terephthalic acid (98+\%) were purchased from Alfa Aesar China Chemicals, TCI Shanghai Chemicals, and Sigma-Aldrich (Shanghai) Chemicals without further purification. All used 20 kinds of solvents were obtained from Beijing Chemicals in purity analysis with names shown in Table 1.

2.2. Gelation Test. All two-component organogels were prepared according to a simple procedure. Firstly, these acids and amino compounds were mixed according to the number matching of intramolecular carboxylic acid and amino groups, respectively. For example, 1,3,5-benzenetricarboxylic acid was mixed with both amino compounds with the molar ratio of $1: 3$, respectively. Then, a weighted amount of binary mixtures and a measured volume of selected pure organic solvent were placed into a sealed glass bottle, and the solution was heated in a water bath with $75^{\circ} \mathrm{C}$ for 20 minutes until the solid was dissolved. Then, the solution was cooled to room temperature in air, and the test bottle was inversed to see if a gel was formed. When the binary mixtures formed a gel by immobilizing the solvent at this stage, it was denoted as "G". For the systems in which only solution remained until the end of the tests, they were referred to as solution (S). When the binary mixtures formed into a few precipitate in some solvent, it was denoted as "PS". The system in which the potential gelator of binary mixtures could not be dissolved even at the boiling point of the solvent was designated as an insoluble system (I). Critical gelation concentration (CGC) refers to the minimum concentration of the gelator for gel formation.

2.3. Characterization Technology. Firstly, these xerogels were prepared by a vacuum pump for $12-24 \mathrm{~h}$ from the as-formed gels under the critical gelation concentration. Then, the dried samples were attached to different substrates, such as copper foil, glass, $\mathrm{KBr}$, or $\mathrm{CaF}_{2}$ slice, for morphological and spectral investigation, respectively. Before SEM measurement, the samples were fixed by conductive adhesive tape and shielded by gold. SEM pictures of the xerogels were taken on a Hitachi S-4800 field emission scanning electron microscopy with the accelerating voltage of $2.0 \mathrm{kV}$. Transmission FT-IR spectra of the xerogels were obtained by a JASCO FT/IR-660 plus spectrophotometer (JASCO Corp., Tokyo, Japan). The measurement results of FT-IR spectra were obtained by average $64 \mathrm{scans}$ and at a resolution of $4 \mathrm{~cm}^{-1}$. The XRD measurement was conducted using a Rigaku D/max 2550PC diffractometer (Rigaku Inc., Tokyo, Japan). The XRD pattern was obtained using $\mathrm{CuK} \alpha$ radiation with an incident wavelength of $0.1542 \mathrm{~nm}$ under a voltage of $40 \mathrm{kV}$ and a current of $200 \mathrm{~mA}$.

\section{Results and Discussion}

3.1. Gelation Behaviors. The gelation performances of all binary mixtures in 20 solvents are tested. The binary mixtures of 2-aminobenzothiazole with terephthalic acid, 1,3,5-benzenetricarboxylic acid, and nitrilotriacetic acid are denoted as SC2, SC3, and SN3, respectively. For the binary mixtures of 2-aminobenzimidazole with these acids, NC2, $\mathrm{NC} 3$, and NN3 are similarly denoted, respectively. The experimental data showed that only the case of SC 3 , the binary mixture of 2-aminobenzothiazole and 1,3,5-benzenetricarboxylic acid could form white opaque organogels in ethanol and acetone, as shown in Figure 1. At the same time, other mixtures were inefficient gelators with the detailed data shown in Table 1. The present experimental results indicated that the change of aromatic/alkyl cores and molecular skeletons in acids and substituted groups in amino derivatives could regulate the gelling abilities of these binary mixtures. It is reported that C3-symmetrical molecules are highly suitable building blocks for the formation of mesophases as well as cylindrically shaped fiber $[21,22]$. In the present case, the C3-symmetric molecular structures and aromatic cores in acids seemed to be more favorable for the gelation of organic solvents than the case with other molecular structures. In addition, the substituted group of benzothiazole showed better gelation properties than benzimidazole part. The reason for the influence of molecular structures on the gelation behaviors could be proposed to the change of the spatial 


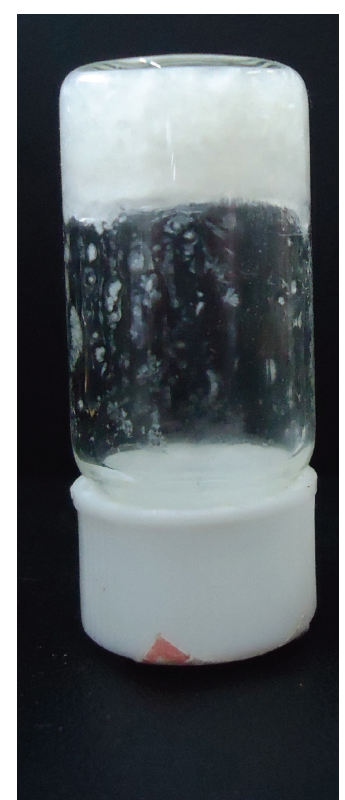

(a)

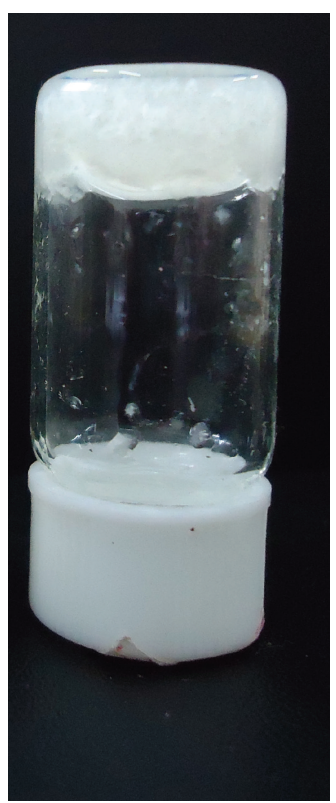

(b)
FIGURE 1: Photographs of SC3 organogels from ethanol (a) and acetone (b), respectively.

conformation and assembly mode of the gelators, which decreased the ability of the gelator molecules to self-assemble into ordered structures, a necessity for forming organized network structures. Furthermore, it should be noted that the organogels of SC3 only form in ethanol and acetone rather than in present other solvents due to the complexed conditions, such as different hydrogen bonding and $\pi-\pi$ interactions, as well as other intermolecular forces between gelators and solvents molecules. So the formation of gels in present system is a cooperative result of many factors.

\subsection{Morphological Investigation. To obtain a visual insight} into the gel microstructures, the typical nanostructures of these gels were studied by SEM technique, as shown in Figure 2. Many researchers have reported that a gelator molecule constructs nanoscale superstructures such as nanofibers, nanoribbons, and nanosheets in a supramolecular gel $[23,24]$. From the present both images, it can be easily investigated that the microstructures of the xerogels of SC3 gels from ethanol and acetone showed similar wrinkleshaped domains composed of sheet-like aggregates with many holes. The similar morphologies suggested similar microstructures and molecular assembly modes for SC3 gels in solvents of ethanol and acetone. The morphologies of the aggregates shown in the SEM images may be also rationalized by considering a commonly accepted idea that highly directional intermolecular interactions, such as hydrogen bonding and/or $\pi-\pi$ interactions, favor formation of belt or fiber structures. It should be noted that the addition of aromatic core in 1,3,5-benzenetricarboxylic acid of SC3 provides the gelators more opportunities in adopting conformations of lower energies, which must weaken the hydrogen-bonding interactions between gelator molecules and result in loss of flexible fine fibrous structure [25].

3.3. Hydrogen Bond Investigation. It is well-known that hydrogen bonding plays an important role in the formation of organogels. At present, in order to further clarify both organogels, we have measured the FT-IR spectra of these binary mixtures in chloroform solution, $\mathrm{KBr}$ pellet, and xerogel forms, as shown in Figure 3. As for the spectrum of SC3 in chloroform solution, as shown in Figure 3(a), two main peaks are observed at 3498,1727 , and $1615 \mathrm{~cm}^{-1}$. These bands can be assigned to the $\mathrm{N}-\mathrm{H}$ stretching, $\mathrm{C}=\mathrm{O}$ stretching of ester, and amide I band, respectively [26]. The spectrum in $\mathrm{KBr}$ pellet is similar to the one above, indicating the obvious characters of functional groups in molecular skeletons. As far as the SC3 xerogels from ethanol or acetone were concerned, these bands shifted to 3396, 1730 , and $1628 \mathrm{~cm}^{-1}$, respectively. The shifts of these bands indicated the formation of different hydrogen bonding in the gel state. Compared with the spectra of xerogels from both different solvents, we found that the spectrum of xerogel from ethanol is similar to that from acetone. This implied that there were similar hydrogen bonding interactions in both xerogels, even though they are from very different solvent systems.

3.4. XRD Investigation. Furthermore, the XRD of both binary xerogels from ethanol and acetone is measured, as shown in Figure 4. The curve for SC3 xerogel from ethanol shows main peaks in the angle region (2 values, $5.20^{\circ}, 11.45^{\circ}, 16.04^{\circ}$, and $18.41^{\circ}$ ) corresponding to $d$ values of $1.70 \mathrm{~nm}, 0.77 \mathrm{~nm}$, $0.55 \mathrm{~nm}$, and $0.48 \mathrm{~nm}$, respectively. In addition, the curve for xerogel in acetone sample shows main peaks in the angle region ( 2 values, $5.25^{\circ}, 11.34^{\circ}, 16.10^{\circ}$, and $18.49^{\circ}$ ) corresponding to $d$ values of $1.68 \mathrm{~nm}, 0.78 \mathrm{~nm}, 0.55 \mathrm{~nm}$, and $0.48 \mathrm{~nm}$, respectively. The corresponding $d$ values in both cases approximately followed a ratio of $1: 1 / 2: 1 / 3: 1 / 4$, suggesting a lamellar-like structure of the aggregates in both gels [27]. This data is in well accordance with the results from morphological investigation. In addition, it is noteworthy that the spacing of the first peak in both curves is very close, which demonstrates that the molecular structures have a great effect on the packing mode of the binary mixtures. Considering the XRD results described above and the hydrogen bonding nature of the organized packing of these binary mixtures as confirmed by FT-IR measurements, a possible packing mode of SC3 organogel in ethanol and acetone was proposed and is schematically shown in Figure 5.

\section{Conclusions}

The gelation behaviors of two-component organogel system based on different acids and aminobenzothiazole derivatives can be regulated by changing molecular skeletons and substituted groups. Only the binary mixture of 2-aminobenzothiazole and 1,3,5-benzenetricarboxylic acid could form organogels in ethanol and acetone. This result 


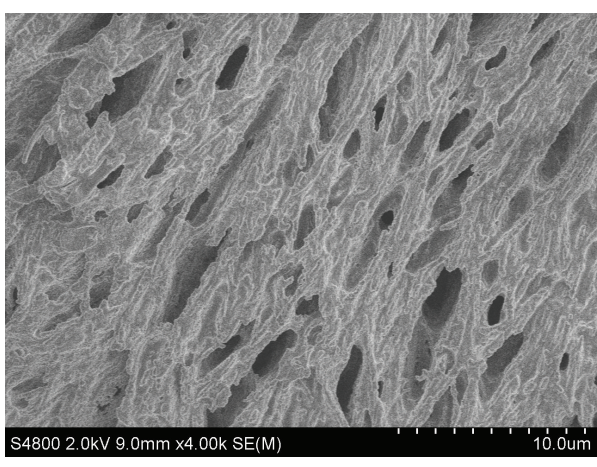

(a)

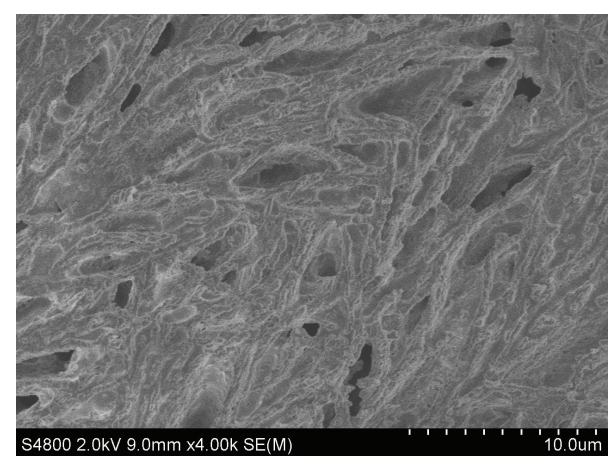

(b)

FIGURE 2: SEM images of xerogels of SC3 gels from ethanol (a) and acetone (b), respectively.

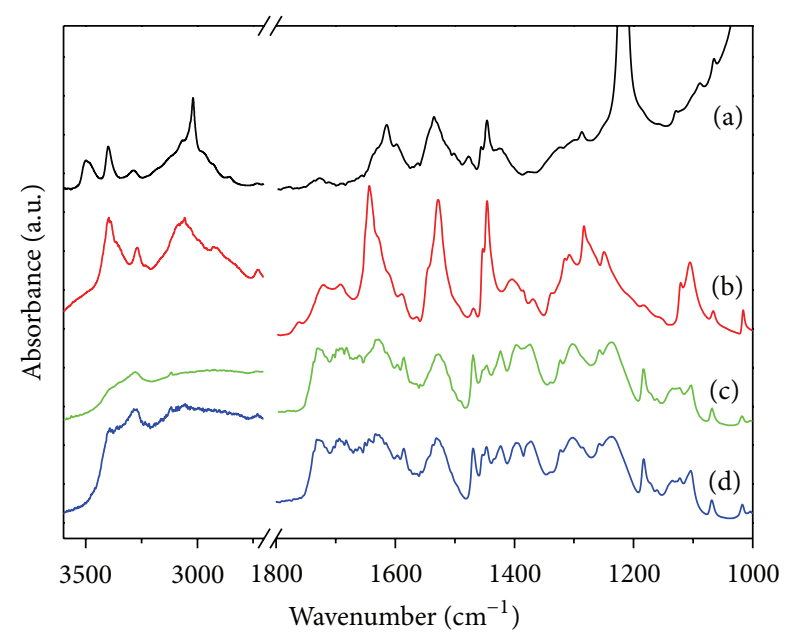

FIGURE 3: FT-IR spectra of SC3 in chloroform solution (a), $\mathrm{KBr}$ pellet (b), and xerogels from gels in ethanol (c) and acetone (d), respectively.

indicated that the C3-symmetric molecular structures and aromatic cores in acids seemed to be more favorable for the gelation of organic solvents than the case with other molecular structures. In addition, the substituted group of benzothiazole showed better gelation properties than benzimidazole part. The microstructures of the xerogels showed similar wrinkle-shaped domains composed of sheetlike aggregates with many holes. FT-IR and XRD studies reveal the hydrogen bonding interaction between the amide of the gelator and lamellar-like structure of the aggregates in both gels. The present investigation is a perspective to provide new clues for the design of new nanomaterials and functional textile materials with special microstructures.

\section{Conflict of Interests}

The authors declare that they have no direct financial relation with the commercial identities mentioned in this paper that might lead to a conflict of interests for any of the authors.

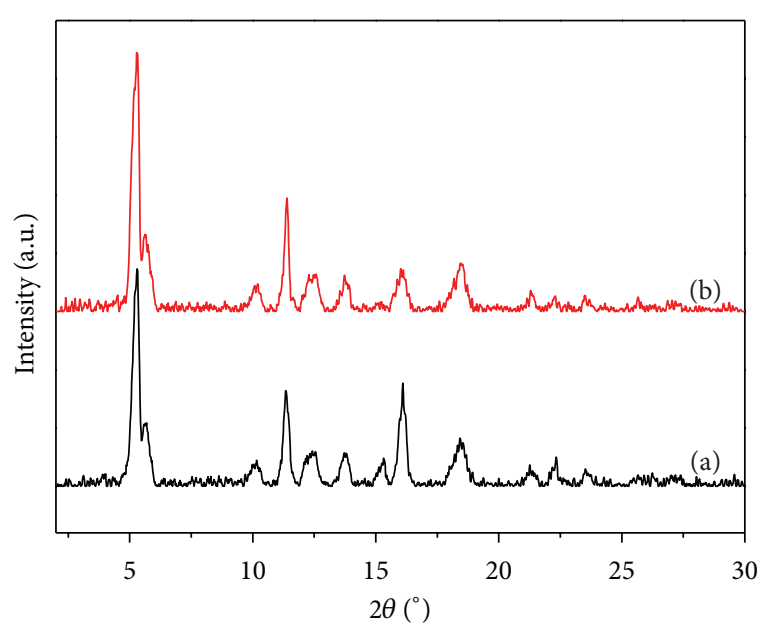

FIGURE 4: X-ray diffraction patterns of xerogels of SC3 gels from ethanol (a) and acetone (b), respectively.

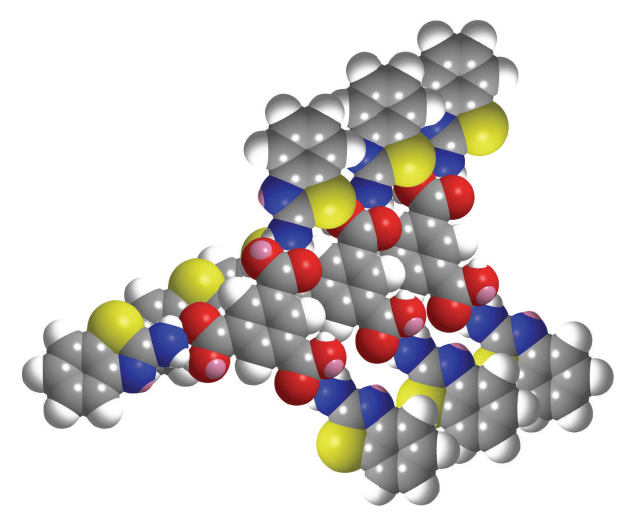

FIGURE 5: A possible assembly mode of SC3 gels from ethanol.

\section{Acknowledgments}

This work was supported by the National Natural Science Foundation of China (50943027) and the Foreign Trade and Economic Development Funds of National Development and Reform Commission of China (2150704). 


\section{References}

[1] P. Deindörfer, T. Geiger, D. Schollmeyer, J. H. Ye, and R. Zentel, "Semicarbazides as gel forming agents for common solvents and liquid crystals," Journal of Materials Chemistry, vol. 16, no. 4, pp. 351-358, 2006.

[2] P. Terech and R. G. Weiss, "Low molecular mass gelators of organic liquids and the properties of their gels," Chemical Reviews, vol. 97, no. 8, pp. 3133-3160, 1997.

[3] S. van der Laan, B. L. Feringa, R. M. Kellogg, and J. Van Esch, "Remarkable polymorphism in gels of new azobenzene bis-urea gelators," Langmuir, vol. 18, no. 19, pp. 7136-7140, 2002.

[4] L. A. Estroff and A. D. Hamilton, "Water gelation by small organic molecules," Chemical Reviews, vol. 104, no. 3, pp. 12011218, 2004.

[5] A. R. Hirst and D. K. Smith, "Two-component gel-phase materials-highly tunable self-assembling systems," Chemistry, vol. 11, no. 19, pp. 5496-5508, 2005.

[6] A. R. Hirst, D. K. Smith, and J. P. Harrington, "Unique nanoscale morphologies underpinning organic gel-phase materials," Chemistry, vol. 11, no. 22, pp. 6552-6559, 2005.

[7] F. Xin, H. Zhang, B. Hao et al., "Controllable transformation from sensitive and reversible heat-set organogel to stable gel induced by sodium acetate," Colloids and Surfaces A, vol. 410, pp. 18-22, 2012.

[8] F. S. Schoonbeek, J. H. van Esch, R. Hulst, R. M. Kellogg, and B. L. Feringa, "Geminal Bis-ureas as gelators for organic solvents: gelation properties and structural studies in solution and in the gel state," Chemistry, vol. 6, no. 14, pp. 2633-2643, 2000.

[9] M. Moniruzzaman and P. R. Sundararajan, "Low molecular weight organogels based on long-chain carbamates," Langmuir, vol. 21, no. 9, pp. 3802-3807, 2005.

[10] B. Escuder, S. Martí, and J. F. Miravet, "Organogel formation by coaggregation of adaptable amidocarbamates and their tetraamide analogues," Langmuir, vol. 21, no. 15, pp. 6776-6787, 2005.

[11] N. M. Sangeetha and U. Maitra, "Supramolecular gels: functions and uses," Chemical Society Reviews, vol. 34, no. 10, pp. 821-836, 2005.

[12] M. de Loos, B. L. Feringa, and J. H. van Esch, "Design and application of self-assembled low molecular weight hydrogels," European Journal of Organic Chemistry, vol. 2005, no. 17, pp. 3615-3631, 2005.

[13] M. George and R. G. Weiss, "Molecular organogels. Soft matter comprised of low-molecular-mass organic gelators and organic liquids," Accounts of Chemical Research, vol. 39, no. 8, pp. 489497, 2006.

[14] M. de Loos, J. H. van Esch, R. M. Kellogg, and B. L. Feringa, "C3symmetric, amino acid based organogelators and thickeners: a systematic study of structure-property relations," Tetrahedron, vol. 63, no. 31, pp. 7285-7301, 2007.

[15] P. Dastidar, "Supramolecular gelling agents: can they be designed?" Chemical Society Reviews, vol. 37, no. 12, pp. 26992715, 2008.

[16] M. Suzuki and K. Hanabusa, "L-lysine-based low-molecularweight gelators," Chemical Society Reviews, vol. 38, no. 4, pp. 967-975, 2009.

[17] P. Mukhopadhyay, Y. Iwashita, M. Shirakawa, S. Kawano, N. Fujita, and S. Shinkai, "Spontaneous colorimetric sensing of the positional isomers of dihydroxynaphthalene in a $1 \mathrm{D}$ organogel matrix," Angewandte Chemie, vol. 45, no. 10, pp. 1592-1595, 2006.
[18] Y. Wang, L. Tang, and Y. Wang, "New hydrogen-bonded supramolecular hydrogels and fibers derived from 1,2,4,5-benzenetetracarboxylic acid and 4-hydroxypyridine," Chemistry Letters, vol. 35, no. 5, pp. 548-549, 2006.

[19] J. Wu, L. Tang, K. Chen, L. Yan, F. Li, and Y. Wang, "Formation of supramolecular hydrogels with controlled microstructures and stability via molecular assembling in a two-component system," Journal of Colloid and Interface Science, vol. 307, no. 1, pp. 280287,2007

[20] T. Jiao, F. Gao, Y. Wang, J. Zhou, F. Gao, and X. Luo, "Supramolecular gel and nanostructures of bolaform and trigonal cholesteryl derivatives with different aromatic spacers," Current Nanoscience, vol. 8, no. 1, pp. 111-116, 2012.

[21] J. J. van Gorp, J. A. J. M. Vekemans, and E. W. Meijer, "C3-symmetrical supramolecular architectures: fibers and organic gels from discotic trisamides and trisureas," Journal of the American Chemical Society, vol. 124, no. 49, pp. 14759-14769, 2002.

[22] V. Percec, M. Glodde, G. Johansson, V. S. K. Balagurusamy, and P. A. Heinev, "Transformation of a spherical supramolecular dendrimer into a pyramidal columnar supramolecular dendrimer mediated by the fluorophobic effect," Angewandte Chemie, vol. 42, no. 36, pp. 4338-4342, 2003.

[23] Y. Wang, L. Tang, and J. Yu, "Investigation on the assembled structure-property correlation of supramolecular hydrogel formed from low-molecular-weight gelator," Journal of Colloid and Interface Science, vol. 319, no. 1, pp. 357-364, 2008.

[24] M. Zinic, F. Vögtle, and F. Fages, "Cholesterol-based gelators," Topics in Current Chemistry, vol. 256, pp. 39-76, 2005.

[25] G. Zhu and J. S. Dordick, "Solvent effect on organogel formation by low molecular weight molecules," Chemistry of Materials, vol. 18, no. 25, pp. 5988-5995, 2006.

[26] T. F. Jiao, Y. J. Wang, Q. R. Zhang, J. X. Zhou, and F. M. Gao, "Regulation of substituent groups on morphologies and self-assembly of organogels based on some azobenzene imide derivatives," Nanoscale Research Letters, vol. 8, no. 160, pp. 1-8, 2013.

[27] M. Kogiso, S. Ohnishi, K. Yase, M. Masuda, and T. Shimizu, "Dicarboxylic oligopeptide bolaamphiphiles: proton-triggered self-assembly of microtubes with loose solid surfaces," Langmuir, vol. 14, no. 18, pp. 4978-4986, 1998. 

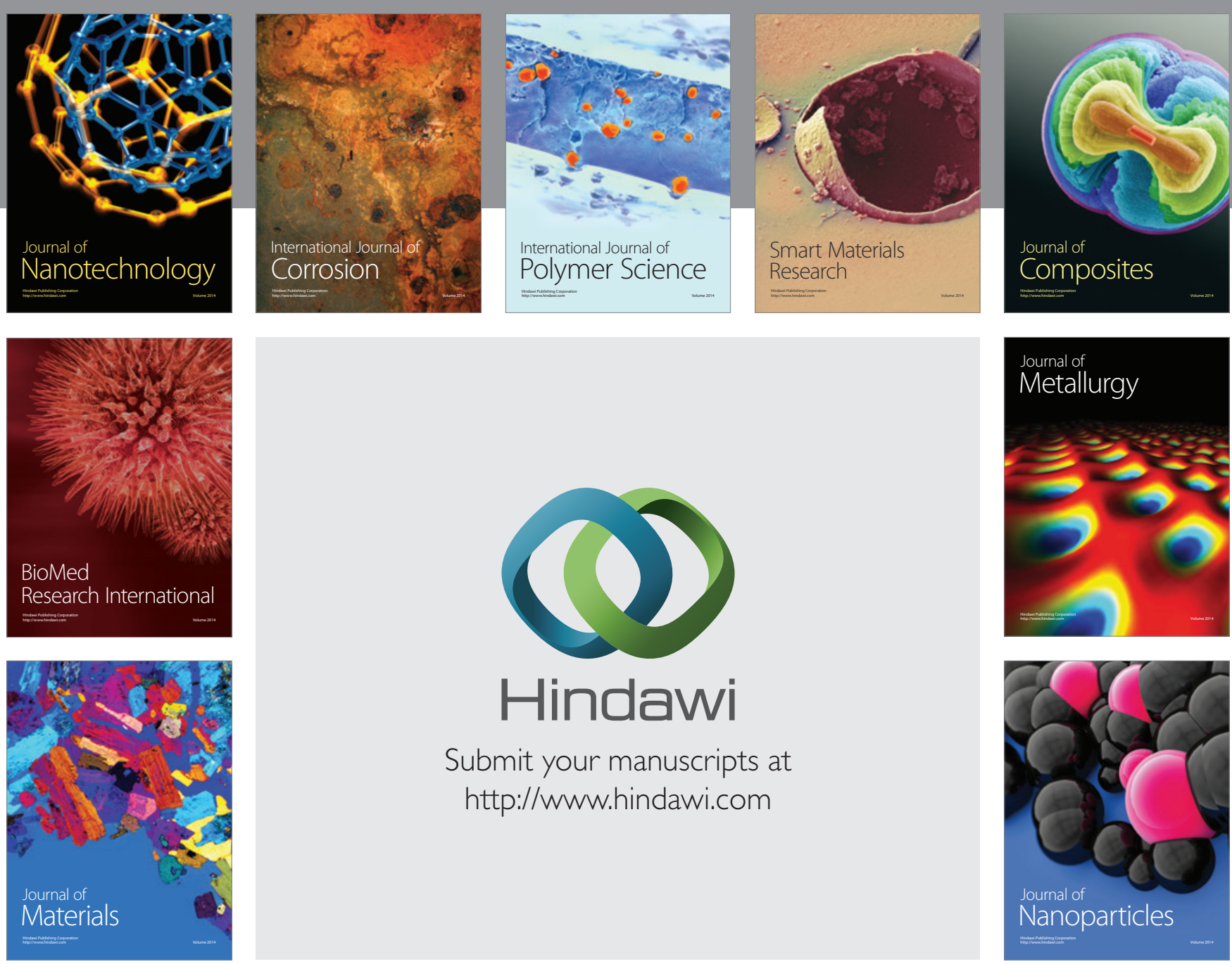

Submit your manuscripts at http://www.hindawi.com
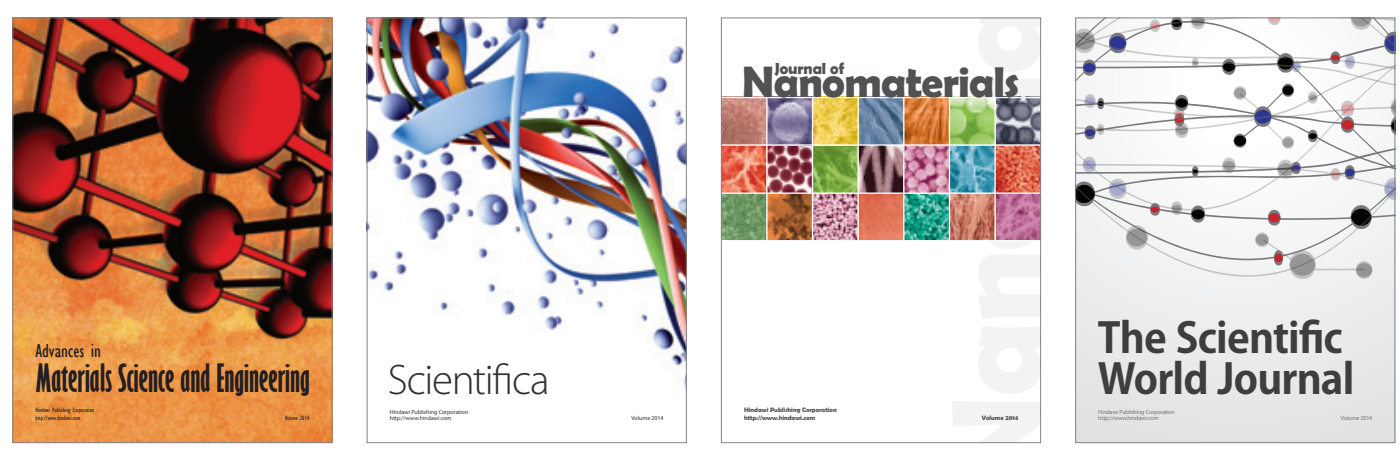

\section{The Scientific World Journal}
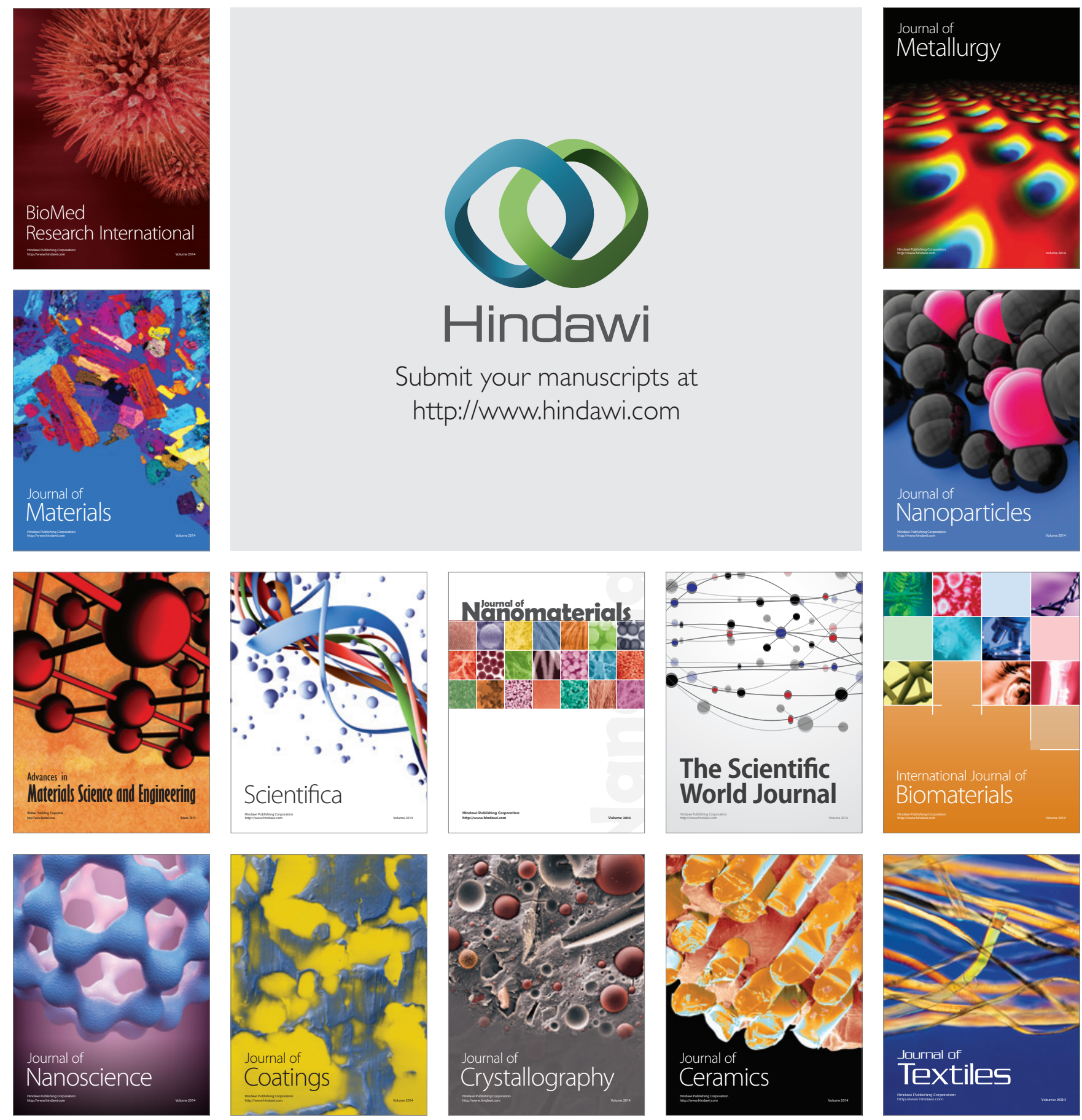